Col I oi dal crystal I i zati on on tilted substrates under gravi tat $\mathrm{i}$ onal fi el ds

\begin{tabular}{|l|l|}
\hline 著者 & $\begin{array}{l}\text { Suzuki Yoshi hi sa, Mori At sushi, Sat o Nasahi de, } \\
\text { Kat suno Hi royasu }\end{array}$ \\
\hline $\begin{array}{l}\text { j our nal or } \\
\text { publ i cat i on t i t l e }\end{array}$ & Journal of Cryst al Grouth \\
\hline vol une & 401 \\
\hline page range & $905-909$ \\
\hline year & $2014-09-01$ \\
\hline URL & ht t p: //hdl . handl e. net /2297/36913 \\
\hline
\end{tabular}




\section{Colloidal crystallization on tilted substrates under gravitational fields}

Yoshihisa Suzuki ${ }^{1, *}$, Atsushi Mori ${ }^{1}$, Masahide Sato $^{2}$, Hiroyasu Katsuno ${ }^{3}$

${ }^{1}$ Institute of Technology and Science, The University of Tokushima, 2-1 Minamijosanjima, Tokushima 770-8506, Japan.

${ }^{2}$ Information Media Center, Kanazawa University, Kakuma-machi, Kanazawa 920-1192, Japan.

${ }^{3}$ Venture Business Laboratory, Nagoya University, Furo-cho, Chikusa-ku, Nagoya 464-8603, Japan.

* Corresponding author. Tel.: +81 88656 7415; Fax: +81 886557025 E-mail address: suzuki@chem.tokushima-u.ac.jp (Y. Suzuki).

\section{ABSTRACT}

We show usefulness of a side wall of a tilted growth container during continuous growth of colloidal crystals under gravitational fields. The side wall works as an effective substrate which enables both enlargement of grains of colloidal crystals and control of crystallographic orientations of the grains. We successfully fabricated large grains of polystyrene face-centered cubic colloidal crystals which align with their $\{111\}$ planes parallel to a side wall by tilting a growth container during centrifugation. Although, without 
tilting a growth container, widths and crystallographic orientations of grains remained unchanged once the widths become asymptotic values, we could increase both widths of grains and population of the grains which aligned with their $\{111\}$ planes parallel to a side wall with terminating grains of the other orientations by tilting the container.

\section{Keywords}

A1. Directional solidification, A1. Growth models, A1. Nucleation, B1. Nanomaterials

\section{Introduction}

A close-packed face-centered cubic ( $\mathrm{fcc}$ ) colloidal crystal is known to be used as a template for the fabrication of an inverse opal with a three dimensional (3D) full photonic band gap (PBG) [1], and a 3D and large close-packed one is easily fabricated by drying almost close-packed (with high volume fraction of particles) large columnar-shaped grains of colloidal crystals which are prepared by centrifugation [2]. By mixing these two techniques, we can fabricate 3D and large inverse opals with 3D full PBG. A 3D and large inverse opal with 3D full PBG is one of the most promising materials which enable us to fabricate very large-scale 3D photonic integrated circuits (PICs) in the interior of the opal or optical computers in the future.

Although such a large-scale 3D PIC has not yet been realized at this stage, small-scale PICs on several semiconductors have already been produced by laser micro-fabrication 
processes [3-4], and laser micro-fabrication techniques of optical waveguides in close-packed colloidal crystals have also been developed [5-6]. In particular, Taton et al. described the importance of enlargement of colloidal crystal for the fabrication of very large-scale PICs [7]. One should note that the enlargement is also useful for mass fabrication of micro PICs by cutting the enlarged single grains as mass fabrication of silicon ( $\mathrm{Si}$ ) microchips is achieved by cutting large $\mathrm{Si}$ wafers and ingots.

Of course, in the case of non-close-packed colloidal crystals, many papers on the fabrication of very large domains of colloidal crystals have been already reported [8-11]. Although the grain size of their crystals are very large, the quality of their crystals is generally fine, and the times for fabrication processes are very short (less than 1 second in the shortest case $[8,10])$, fabrication of inverse opals from their crystals is difficult.

Although widening of columnar grains has been also well achieved with the control of nucleation of grains by centrifugation [2, 12], further enlargement requires additional developments of growth methods. Several grains were nucleated all together at the bottom of a flat-bottomed container in these studies. Competitions of growth between adjacent grains occur immediately after the nucleation, and then the average width of the grains soon approaches an asymptotic value. Recently our group successfully widened columnar grains using an inverted-triangle-inner-shaped container [13]. This success is mainly due to the initial limitation of the number of grains and subsequent widening of the grains via the inverted-triangle-inner-shaped bottom. Additionally, from a different viewpoint, we could continuously widen the bottom area of columnar grains owing to a sloped wall of the inverted-triangle-inner-shaped bottom, while we could not widen the area in the case of a 
flat-bottomed container. However, we have not controlled the crystallographic orientation of a grain at all, while the control of the orientation is a prerequisite for device applications.

To control the orientation, we focus that fcc colloidal crystals usually crystallize with one of their $\{111\}$ faces parallel to flat substrates [14-15]. If we can efficiently use this preference for the enlargement of grains and control of their orientations, centrifugation method becomes much more important one.

In this study, to enlarge grains and to control their orientations, we try to utilize a side flat wall of a growth container as the flat substrate by tilting the container from the rotational plane of a centrifugation apparatus, since a tilted wall is useful for widening the bottom area of columnar grains, and one of the $\{111\}$ faces of an fcc colloidal crystal preferentially aligns parallel to a flat wall.

\section{Experimental}

\subsection{Sample and apparatus}

Water suspensions of polystyrene particles (Duke Scientific Co. Ltd., 5020A, diameter of particles $d=200 \mathrm{~nm}$ ) were used without further purification.

A thin growth container $(0.1 \mathrm{~mm}$ thick, $9.0 \mathrm{~mm}$ wide, and $50.0 \mathrm{~mm}$ long $)$ was cut and a piece of Si plate (1.0 mm thick, a (001) oriented wafer) was adhered carefully to the cut end of the cell using a silicone adhesive (Fig. 1(a)). Suspensions of particles were injected into growth containers and bubbles inside were removed completely by applying centrifugation.

Gravitational force was applied by centrifugation. Growth containers were set onto a sample holder, and the rotational shaft of the holder was inserted into the chuck hole of a 
stirrer (Iuchi, SM101). We set growth containers onto the holder in following two ways. One was horizontal setting, and the other was tilted setting as shown in Fig. 1(b). In the former case, the container was set horizontally, and the orientation of centrifugal acceleration was perpendicular to the bottom Si substrate. On the other hand, in the latter case, the container was tilted from the rotational plane, and the side wall of the container became the substrate onto which grains grow. The angle between the side wall of the container and the rotational plane in the tilted setting was $\sim 35^{\circ}$.

\section{2. Principle of growth methods}

Growth processes of colloidal crystals in the horizontal and tilted settings are schematically shown in Fig. 2(a) and (b), respectively. In the case of the horizontal setting (Fig. 2(a)), at first, nucleation of grains occurs at a bottom Si substrate at once, and then, subsequent growth of columnar grains continues. Most of the grains are those nucleated at the bottom wall with their $\{111\}$ faces parallel to the bottom wall, while some grains (red particles in Fig. 2) adjacent to side walls grow with their $\{111\}$ faces parallel to the side walls. In the case of the tilted setting (Fig. 2(b)), nucleation occurs at the edge between the upper side wall and the Si bottom substrate, and then, at least two grains (red and white particles in Fig. 2(b)) start to grow with their $\{111\}$ faces parallel to a side wall and Si bottom substrate as shown in the leftmost picture of Fig. 2(b). Both the side wall and Si bottom substrate are used as tilted substrates, and thus, the growth of grains is accompanied by widening of bottom areas of columnar grains as shown in the middle picture of Fig. 2(b). In Fig. 2(b), the grain which is grown from the bottom wall (white particles in Fig. 2(b)) 
reaches the other side wall. Therefore, after this, the width of this grain becomes narrow, and then, the grain stops growing as shown in the rightmost picture of Fig. 2(b). At the same time, the grain which is grown from the upper side wall (red particles in Fig. 2(b)) occupies whole area, and it continues to grow afterward (Fig. 2(b), the rightmost picture). At this stage, the size of the grain which is grown from the upper side wall is efficiently enlarged, and the orientation of the grain becomes uniform as one of $<111>$ directions of the grain perpendicular to the side wall.

\section{3. Characterization}

Transmission polarization and reflection bright field microscopic observations of colloidal crystals and stacking disorders in grains were conducted (Olympus Co., Ltd., VANOX) perpendicular to side walls. Orientations of grains were determined by ultraviolet-visible (UV-VIS) reflection spectroscopy (Ocean Optics Co., Ltd., USB4000).

\section{Results and discussion}

\subsection{Horizontal setting}

Figure 3(a) shows a merged transmission cross-polarized image of a colloidal polycrystal which grew in a thin growth container set on a sample holder horizontally by centrifugation as shown in Fig. 1(b). After 13 days centrifugation at $84 \mathrm{G}$ at the bottom of the growth container, columnar grains grew without significant widening of their asymptotic widths. This asymptotic behavior is mainly because (1) widths of grains are proportional to the 
square root of height of grains and inversely proportional to the number of initial nuclei as a result of geometrical selection (Kolmogorov's relation) [12], and (2) no additional nucleation occurs in front of growth interface of columnar grains owing to relatively low centrifugal acceleration $(84 \mathrm{G})$ in this system (small and light polystyrene particles $(d=200$ $\mathrm{nm})$ ). Therefore, in the horizontal setting, to obtain larger grains suppression of the nucleation at the bottom is indispensable, although the control of nucleation which occurs at once at the bottom is usually difficult.

In Fig. 3(a), most of grains show various retardation colors. In the case of fcc colloidal crystals, transmission cross-polarized images perpendicular to their $\{111\}$ planes show complete extinction, while those perpendicular to the other lattice planes show various colors of retardation [16]. Many grains show relatively bright retardation colors in Fig. 3(a), while a few grains exhibit extinction in the horizontal setting. Thus, most of grains do not align with their $\{111\}$ planes parallel to the side wall. This is due to the fact that most of grains nucleate with their $\{111\}$ face parallel to the bottom Si substrate, since side walls are perpendicular to the bottom Si substrate; one $\{111\}$ plane of an fcc crystal cannot be perpendicular to the other $\{111\}$ planes geometrically. In addition, there are many striations in columnar grains (Fig. 3(a)). Monovoukas and Gast observed polystyrene colloidal crystals using a transmission polarization microscope, and found similar striations [16]. They concluded that the striations indicate twinned structures in a columnar grain. In our case, there are striations of repeated retardation color pairs (dark and bright retardation colors). Monovoukas and Gast showed that the retardation colors of twinned area change all together by rotating the crystals. For instance, in this study, dark colored areas repeating 
in a columnar grain change their colors brighter all together during a rotation of the cell. On the contrary, at the same time, bright colored areas next to the dark colored areas change their colors darker all together. From these results, we conclude the striations observed in a transmission cross-polarized image correspond to twinned structures of colloidal crystals. Therefore, a uniform-colored area between twin boundaries probably indicates stacking-disorder-free area. Largest uniform-colored area in Fig. 3(a) is about $1.5 \times 0.5$ $\mathrm{mm}^{2}$, and thickness of inner cell is $0.1 \mathrm{~mm}$. This size is still large as such high-quality nearly close-packed colloidal crystals which have been so far reported. Although Davis et al. earlier observed stacking faults by scanning electron microscopy (SEM), and they asserted that the stacking faults resulted in the columnar looking of the crystals [17], it is suspicious, since intervals of their columnar boundaries were much larger than those of stacking faults shown in SEM micrographs, and the shapes of columnar boundaries were not straight as usual stacking disorders, which are shown in Fig. 3(a).

\subsection{Tilted setting}

Figure 3(b) shows a merged transmission cross-polarized image of a colloidal polycrystal which grew in a thin growth container set on the sample holder in the tilted setting as shown in Fig. 1(b). After 20 days centrifugation at $21 \mathrm{G}$ at the bottom of the container, dark-colored grains were mainly obtained, while a few grains near the container bottom show various bright retardation colors. Dark-colored grains showed extinction, while complete extinction was not observed with a simple polarization microscope setup in this study. The grains (grains exhibiting extinction) were those aligned with their $\{111\}$ planes 
parallel to the side wall as described in section 3.1 [16]. These grains grew wider and terminated the other grains with their $\{111\}$ plane nonparallel to the side wall. The volume of the largest grain in Fig. 3(b) is about $1.0 \times 2.0 \times 0.1 \mathrm{~mm}^{3}$.

Orientations of the grains exhibiting extinction are confirmed by reflection bright field microscopy and UV-VIS spectroscopy. Figure 4(a) shows a merged reflection bright-field image of the same colloidal polycrystal which is shown in Fig. 3(b). The grains exhibiting extinction in Fig. 3(b) show almost uniform green color which indicates the color of Bragg diffraction from $\{111\}$ planes parallel to the upper side wall of the cell. A UV-VIS spectrum at the red circle marked in Fig. 4(a) is shown in Fig. 4(b), and peak wavelength $\lambda$ is estimated to be $\lambda=537.3 \mathrm{~nm}$ by fitting with a Gaussian curve. This peak wavelength well corresponds to $\lambda=531.2 \mathrm{~nm}$, which is calculated as a Bragg wavelength from $\{111\}$ planes of an fcc colloidal crystal using a volume fraction of a colloidal crystal $\phi_{\mathrm{c}}=0.55$, particle diameter $d=200 \mathrm{~nm}$, average refractive index $n=n_{\mathrm{p}} \phi_{\mathrm{c}}+n_{\mathrm{w}}\left(1-\phi_{\mathrm{c}}\right)=1.59 \times 0.55+1.33$ $\times 0.45=1.47$, where $n_{\mathrm{p}}$ is refractive index of polystyrene particles and $n_{\mathrm{w}}$ is that of water. Thus, dark-colored grains in Fig. 3(b), which are effectively enlarged in the tilted setting, align with their $\{111\}$ planes parallel to the side wall of the cell.

This enlargement is mainly due to the preference of the $\{111\}$ alignment of fcc colloidal crystals parallel to flat substrates. Although geometrical selection is well known to enlarge the width of columnar grains [12], typical width of geometrically selected grains grown from the bottom Si substrate in this study is up to $0.5 \mathrm{~mm}$ at most. On the other hand, in the tilted setting, the width becomes much larger (up to $1 \mathrm{~mm}$ ), owing to obvious widening of $\{111\}$ aligned grains. This widening is due to the preferential alignment of the $\{111\}$ 
planes parallel to a flat wall [14-15].

In this study, we set the angle of tilting growth containers $\sim 35^{\circ}$ from rotational plane of a sample holder. If a grain grows with its (111) face parallel to the side wall and with its $<11 \overline{2}>$ direction parallel to the longitudinal direction of a growth cell, and if the angle is exactly equal to $35.26^{\circ}$, which is the angle between the (111) face and (110) face of an fcc crystal, its growth interface will become its (001) face. This face is very useful to suppress the induction of stacking disorders, since there is unique way to stack particle layers onto the (001) face of an fcc crystal, while there are two ways (uncertainty) to place particles onto the (111) face [18]. Therefore, if we change the angle and crystallographic plane of a growth interface, the size and orientation will be change. To find out better conditions by changing the tilt angle is remained as future work. In addition, if we use a thicker growth container, thicker grains will be obtained.

Although further enlargement of a grain will be soon achieved as described above, the disappearance of all stacking disorders in the grain (Fig. 3(a) and 3(b)) has not been achieved yet. To anneal out the stacking disorders from the obtained grains, we have been focusing that the cross-coupling term between an elastic field due to downward gravity and that due to a Shockley partial dislocation in crystals results in the movement of the dislocation upward [19]. Actually, Monte Carlo simulations of growth of hard-sphere crystals under gravitational fields clearly showed that applications of gravitational fields are useful to reduce stacking disorders in the crystals [20]. Recently, the reduction of stacking disorders in silica colloidal crystals by centrifugation was experimentally achieved [21]. The disappearance of all stacking disorders in a grain is what we are aiming for as future 
work.

We stress here that a centrifugation method was adopted not for the acceleration of crystallization processes but for the precise control of nucleation rates in this study. Although, for a real application, a rapid and facile method is required, and a long time period of our method seems to be a drawback, at this stage, as far as we know, no other methods have succeeded to fabricate a close-packed, $\{111\}$ oriented, stacking-disorder-free, bulk $\left(\sim 1.0 \times 2.0 \times 0.1 \mathrm{~mm}^{3}\right)$, and single colloidal crystal (grain) as we have fabricated here. Of course, we can shorten the period for the fabrication of sufficiently large and high quality grains by increasing the rotational speed of a stirrer gradually and keeping the centrifugal acceleration at a growth interface an appropriate value. Ideally, as long as centrifugal acceleration at a growth interface is lower than the critical value for nucleation of additional grains, we can increase the acceleration, and shorten the period for the fabrication significantly. We have a plan to conduct such trials by the use of a programmable centrifugation apparatus in the future.

\section{Conclusions}

In this paper, we confirmed the usefulness of a side wall of a tilted growth container for colloidal crystallization by centrifugation. Our key findings are as follows.

1) In the horizontal setting, width of grains became almost constant (at most $\sim 0.5 \mathrm{~mm}$ ). A transmission cross-polarized image of grains showed twinned structures, and 
largest stacking-disorder-free area is $\sim 1.5 \times 0.5 \mathrm{~mm}^{2}$.

2) In the tilted setting, grains exhibiting extinction preferentially grew and terminated the other bright-colored grains. We obtained much larger grains than those in the horizontal setting $\left(\sim 1.0 \times 2.0 \times 0.1 \mathrm{~mm}^{3}\right.$ at largest $)$.

3) We measured the orientation of grains exhibiting extinction using a reflection bright-field microscope and a UV-VIS spectrometer. All the grains aligned with one of their $\{111\}$ planes parallel to a side wall of a growth container.

\section{Acknowledgements}

This work was partially supported by Grants in Aid (No. 24656016 (Y. S.)) of Scientific Research of the Ministry of Education, Culture, Sports, and Technology Japan.

\section{References}

[1] A. Blanco, E. Chomski, S. Grabtchak, M. Ibisate, S. John, S.W. Leonard, C. Lopez, F. Meseguer, H. Miguez, J.P. Mondia, G.A. Ozin, O. Toader, H.M. van Diel, Large-scale synthesis of a silicon photonic crystal with a complete three-dimensional bandgap near 1.5 micrometres, Nature 405 (2000) 437-440.

[2] Y. Suzuki, A. Mori, T. Fujiwara, K. Tamura, Precise characterization of grain 
structures, stacking disorders, and lattice disorders of a close-packed colloidal crystal, Journal of Crystal Growth 322 (2011) 109-113.

[3] H. C. Nguyen, Y. Sakai, M. Shinkawa, N. Ishikura, T. Baba, $10 \mathrm{~Gb} / \mathrm{s}$ operation of photonic crystal silicon optical modulators, Optics Express 19 (2011) 13000-13007.

[4] L. Thylén, M. Qiu, S. Anand, Photonic Crystals-A Step towards Integrated Circuits for Photonics, ChemPhysChem 5 (2004) 1268-1283.

[5] W. Lee. S. A. Pruzinsky P. V. Braun, Multi-Photon Polymerization of Waveguide Structures Within Three-Dimensional Photonic Crystals, Advanced Materials 14 (2002) 271-274.

[6] Y. Jun, C. A. Leatherdale, D. J. Norris, Tailoring air defects in self-assembled photonic bandgap crystals, Advanced Materials 17 (2005) 1908-1911.

[7] T. A. Taton, D. J. Norris, Device physics: Defective promise in photonics, Nature 416 (2002) 685-686.

[8] T. Sawada, Y. Suzuki, A. Toyotama, N. Iyi, Quick fabrication of gigantic single-crystallinze colloidal crystals for photonic crystal applications, Japanese Journal of Applied Physics 40 (2001) L1226-L1228.

[9] J. Yamanaka, M. Murai, Y. Iwayama, M. Yonese, K. Ito, T. Sawada, One-directional crystal growth in charged colloidal silica dispersions driven by diffusion of base, Journal of the American Chemical Society 126 (2004) 7156-7157.

[10] T. Kanai, T. Sawada, A. Toyotama, K. Kitamura, Air-pulse-drive fabrication of photonic crystal films of colloids with high spectral quality, Advanced Functional 
Materials 15 (2005) 25-29.

[11] A. Toyotama, J. Yamanaka, M. Yonese, T. Sawada, F. Uchida, Thermally driven unidirectional crystallization of charged colloidal silica, Journal of the American Chemical Society 129 (2007) 3044-3045.

[12] Y. Suzuki, T. Sawada, K. Tamura, Colloidal crystallization by a centrifugation method, Journal of Crystal Growth 318 (2011) 780-783.

[13] K. Hashimoto, A. Mori, K. Tamura, Y. Suzuki, Enlargement of grains of silica colloidal crystals by centrifugation in an inverted-triangle internal-shaped container, Japanese Journal of Applied Physics 52 (2013) 030201-1-3.

[14] M. Heni, H. Löwen, Interfacial free energy of hard-sphere fluids and solids near a hard wall, Physical Review E 60 (1999) 7057-7065.

[15] J. P. Hoogenboom, D. Derks, P. Vergeer, A. van Blaaderen, Stacking faults in colloidal crystals grown by sedimentation, Journal of Chemical Physics 117 (2002) $11320-11328$.

[16] Y. Monovoukas, A. P. Gast, A study of colloidal crystal morphology and orientation via polarizing microscopy, Langmuir 7 (1991) 460-468.

[17] K. E. Davis, W. B. Russel, W. J. Glantschnig, Disorder-to-order transition in settling suspensions of colloidal silica: x-ray measurements, Science 245 (1989) 507-510.

[18] A. van Blaaderen, R. Ruel, P. Wiltzius, Template-directed colloidal crystallization, Nature 385 (1997) 321-324.

[19] A. Mori, Y. Suzuki, Interplay between elastic fields due to gravity and a partial dislocation for a hard-sphere crystal coherently grown under gravity: driving force for 
defect disappearance, Molecular Physics 108 (2010) 1731-1738 .

[20] A. Mori, Y. Suzuki, S. Yanagiya, T. Sawada, K. Ito, Shrinking stacking fault through glide of the Shockley partial dislocation in hard-sphere crystal under gravity, Molecular Physics 105 (2007) 1377-1383.

[21] Y. Suzuki, J. Endoh, A. Mori, T. Yabutani, K. Tamura, Gravitational annealing of colloidal crystals, Defect and Diffusion Forum 232-235 (2012) 555-558.

\section{Figure captions}

Fig. 1. Schematic illustrations of (a) a thin growth container for growth and observation and (b) setting geometries of the containers and a sample holder.

Fig. 2. Schematic illustrations of growth processes in (a) horizontal setting and (b) tilted setting. Red particles indicate those of grains which nucleate on side walls.

Fig. 3. Merged transmission cross-polarized images of obtained colloidal crystals. The images are perpendicular to side walls of flat capillary cells. (a): horizontal setting (b): tilted setting White arrows indicate growth directions. Scale bar represents $1 \mathrm{~mm}$. The hatched region indicates single grain of the crystal.

Fig. 4. (a): A merged reflection bright-field image of a colloidal polycrystal (Fig. 3 (b)). 
Scale bar represents $1 \mathrm{~mm}$. A red circle indicates a position where a spectrum is attained.

(b): Spectrum data obtained at the red circle position. A peak wavelength of the spectrum is $537.3 \mathrm{~nm}$. 


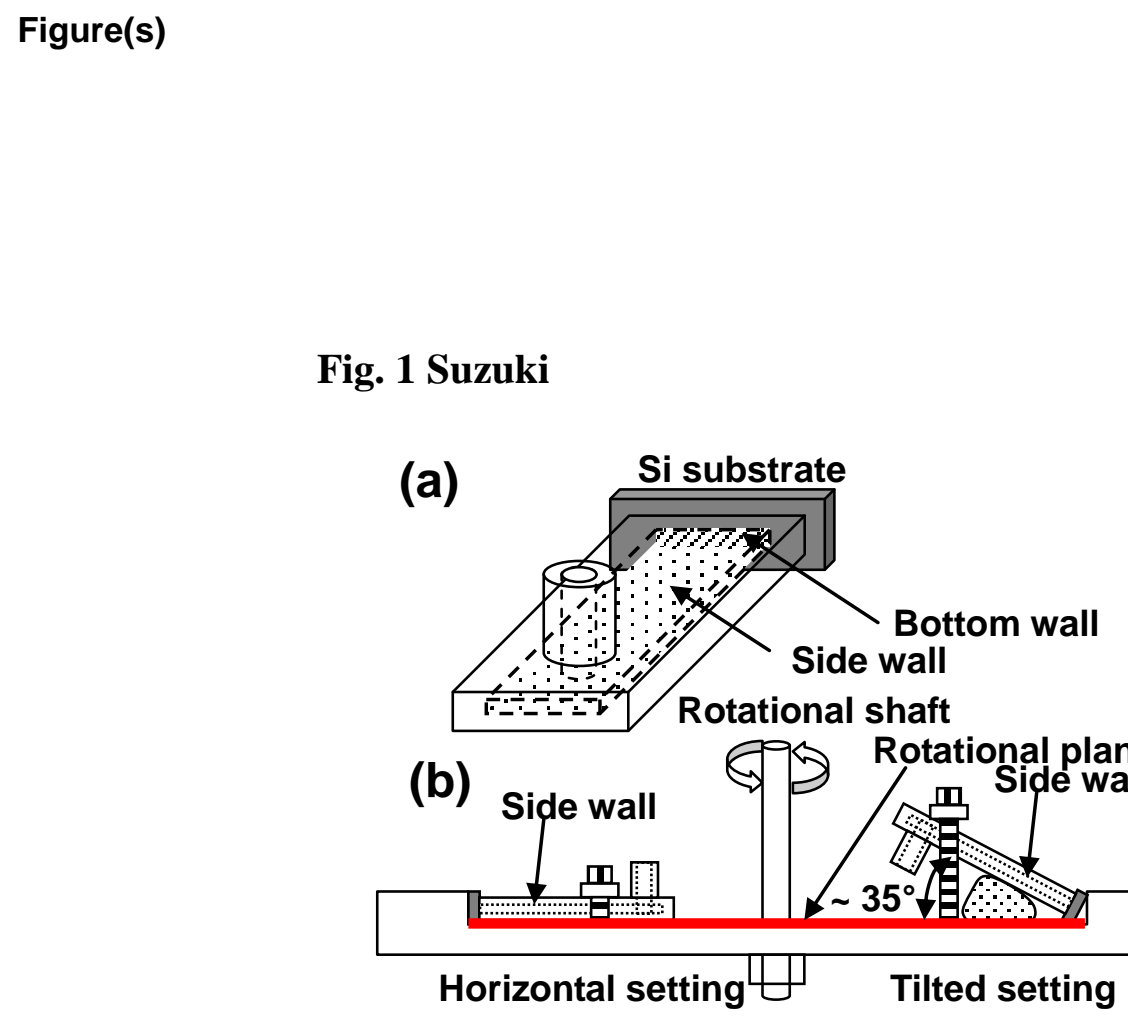
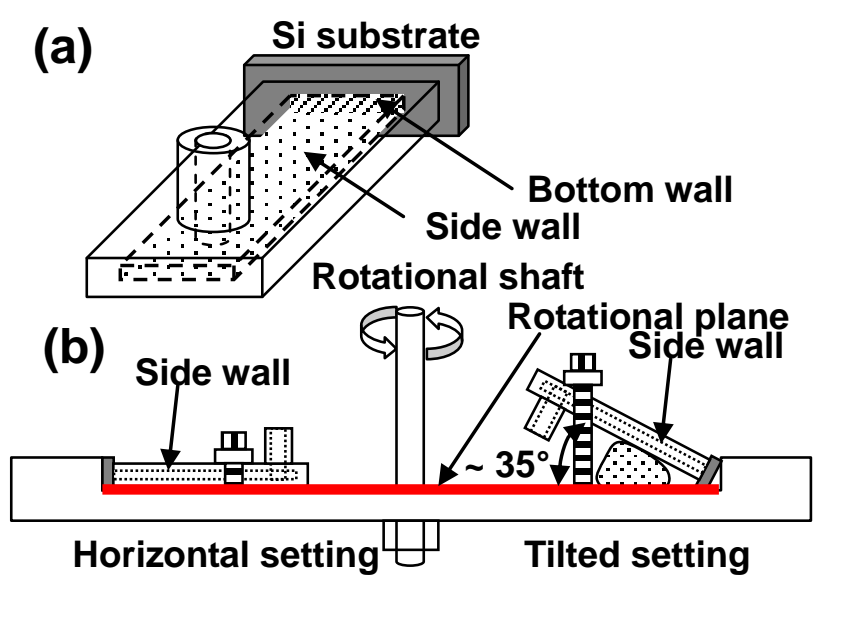

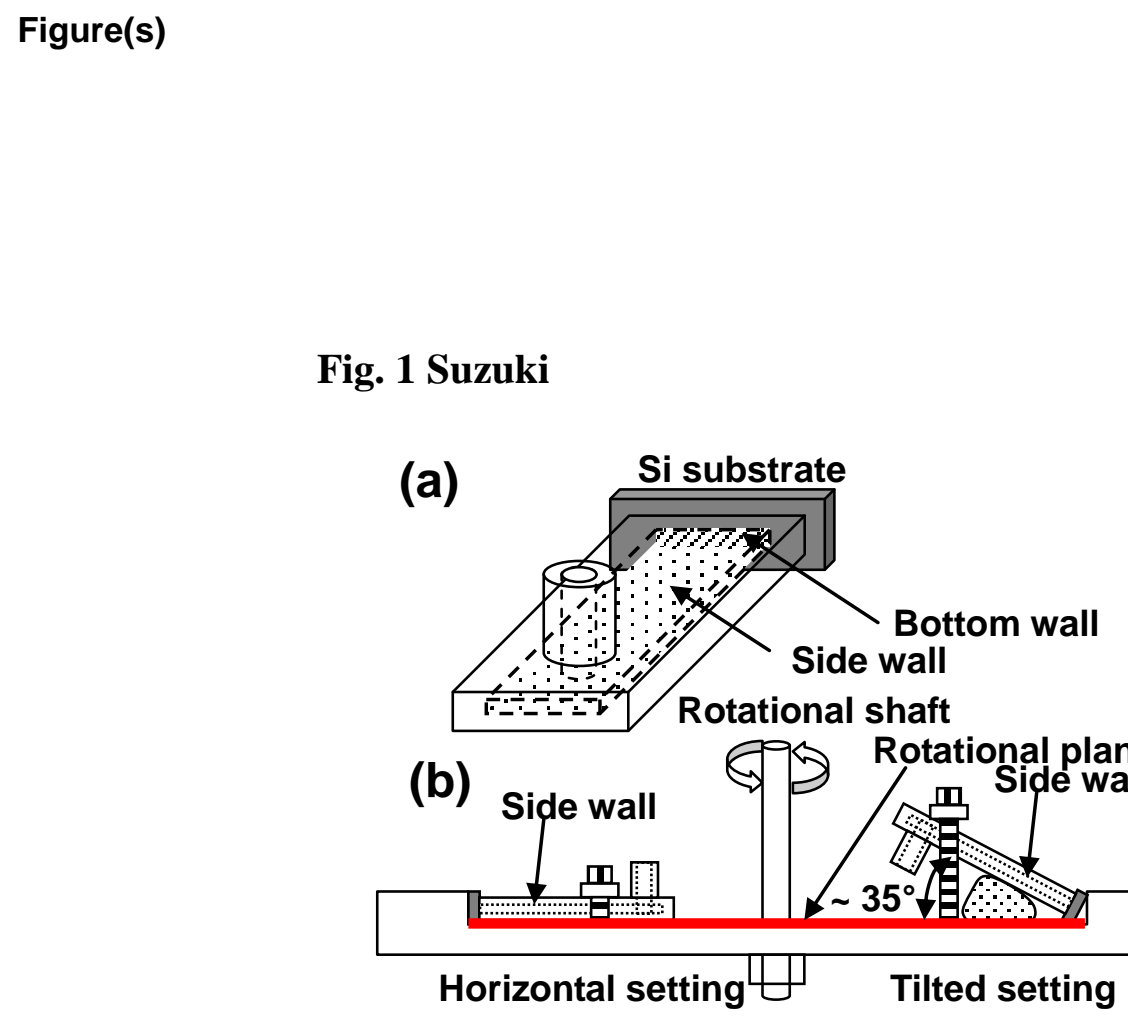
(1)

(n)

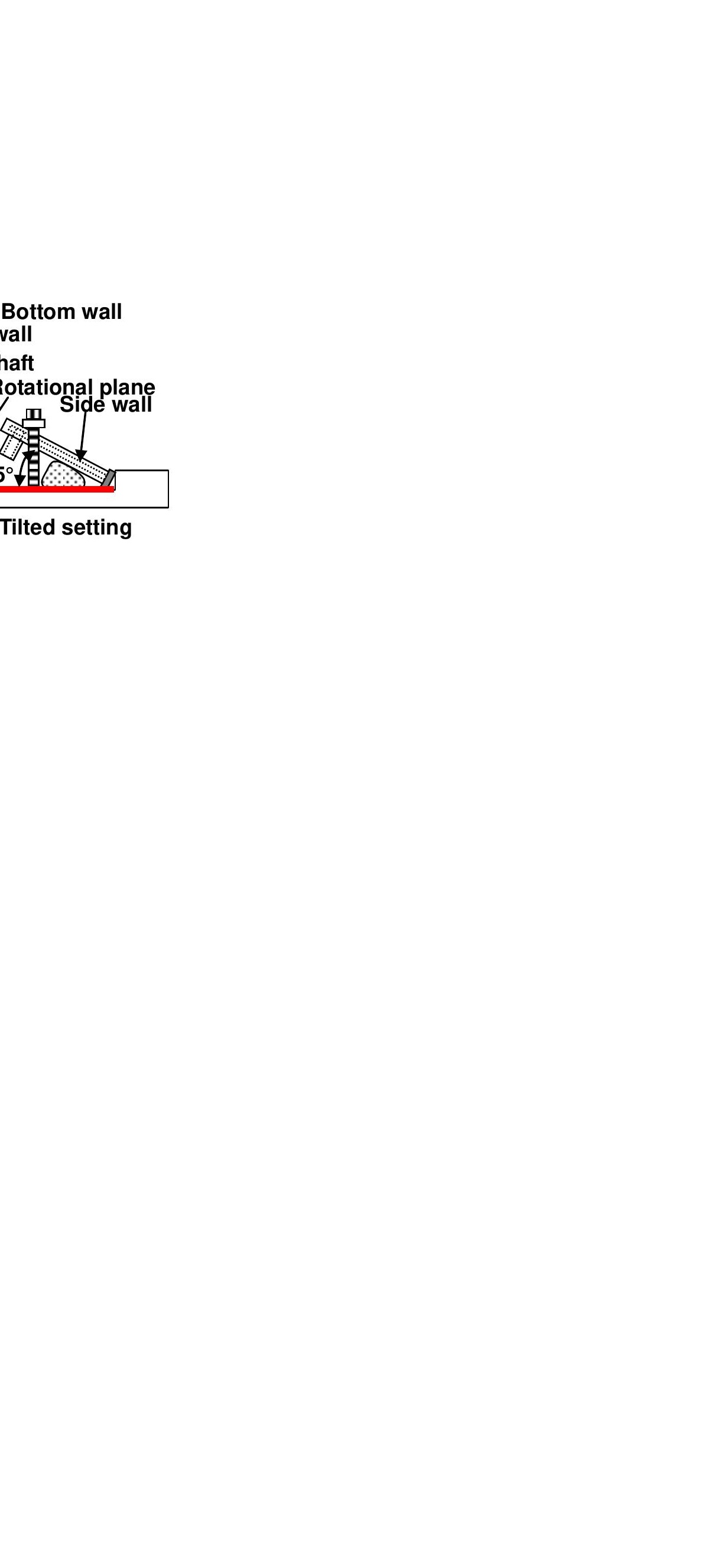


Fig. 2 Suzuki

(a) Horizontal setting
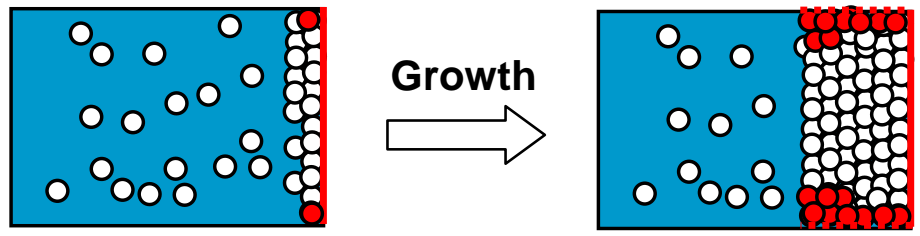

(b) Tilted setting
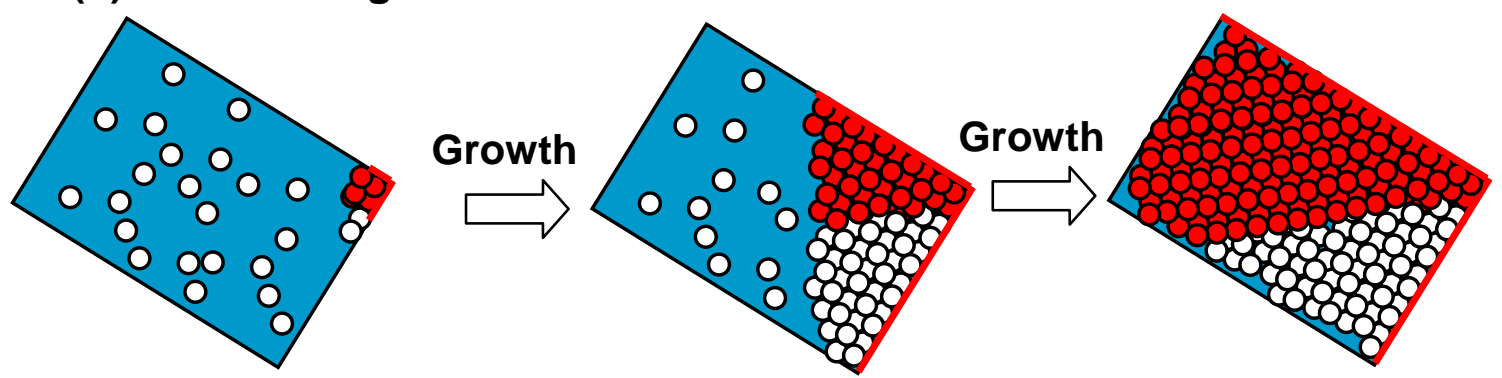
Fig. 3. Suzuki
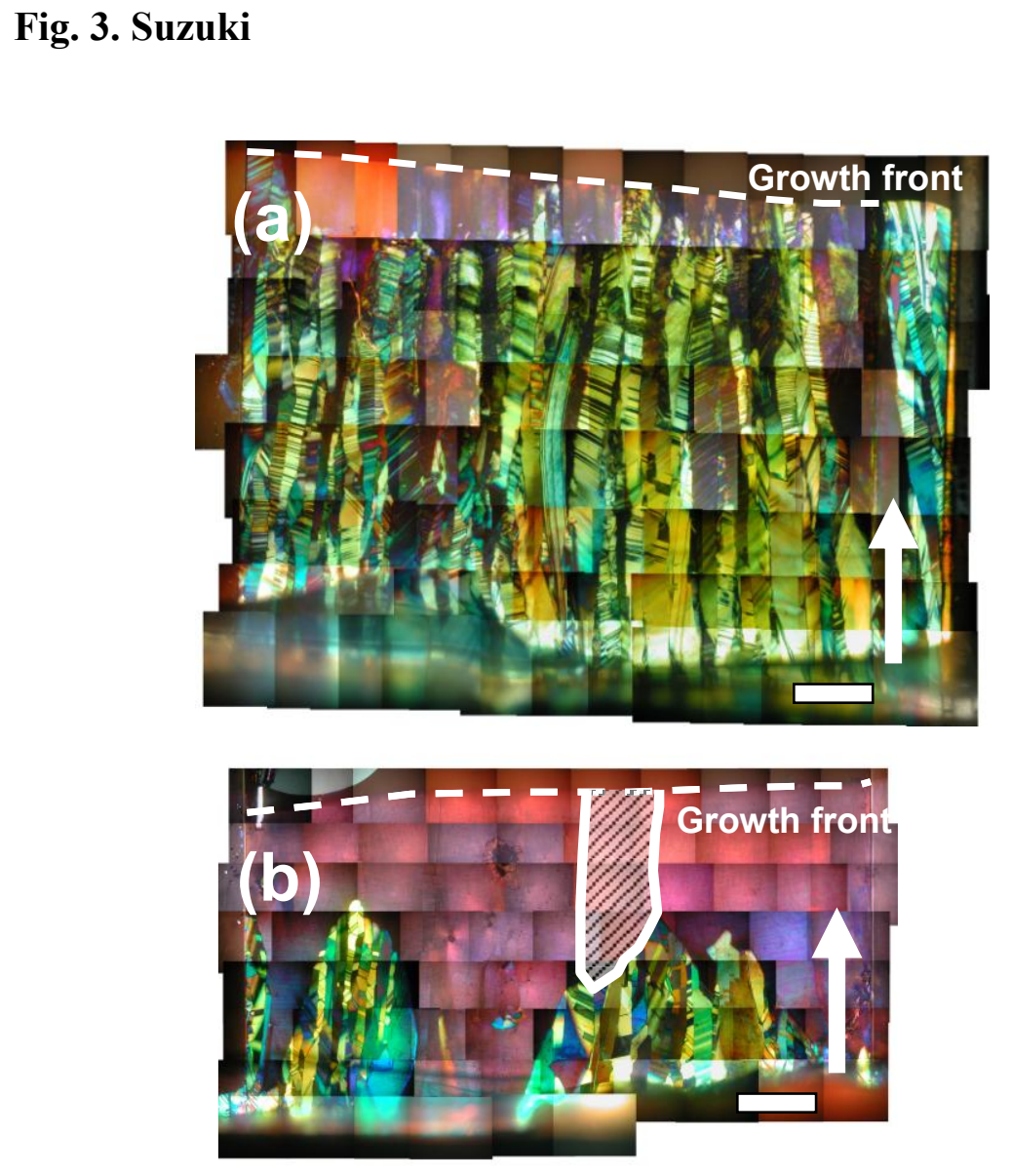

Figure(s)

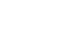

\section{.}

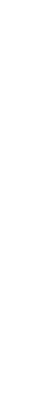


Fig. 4. Suzuki
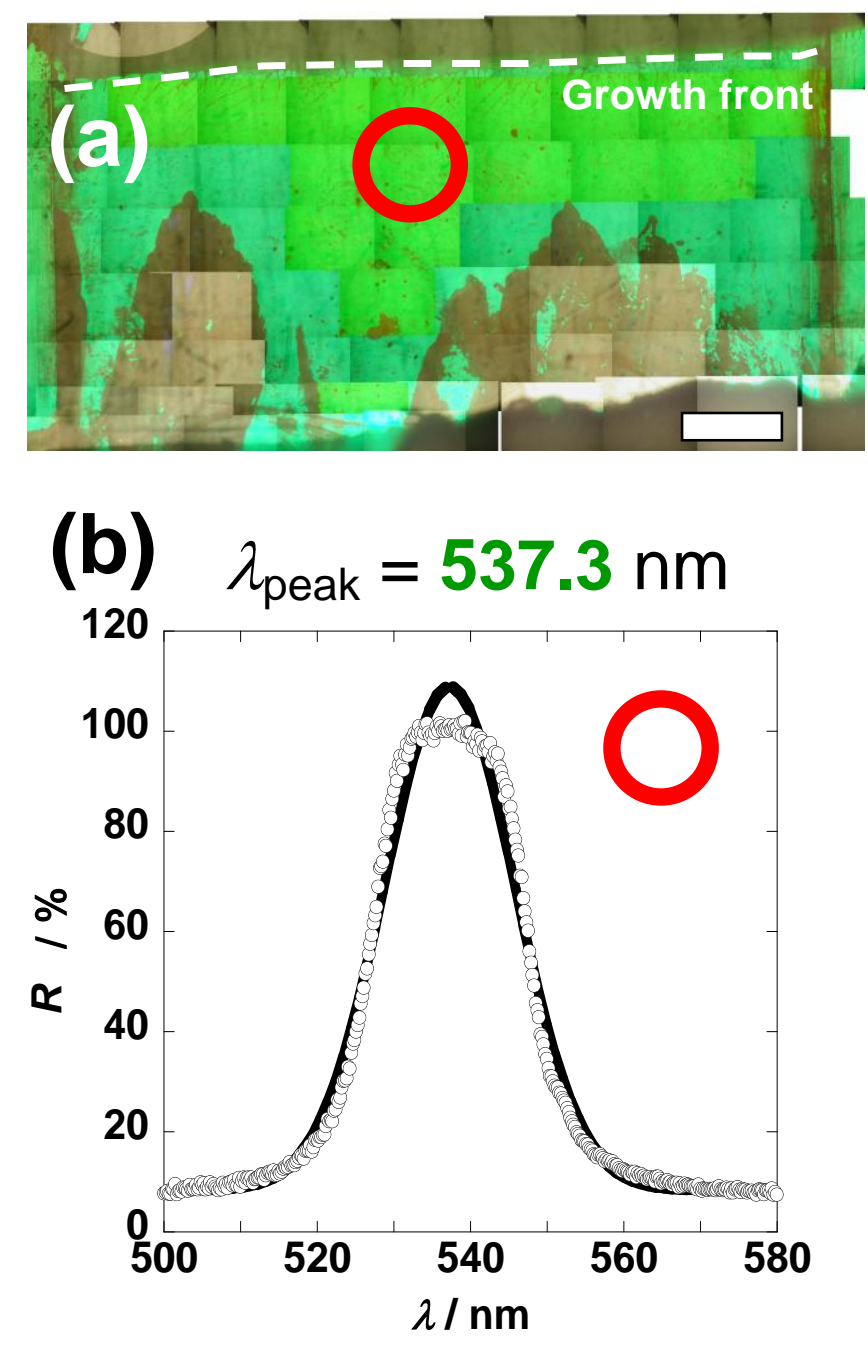

Figure(s) 